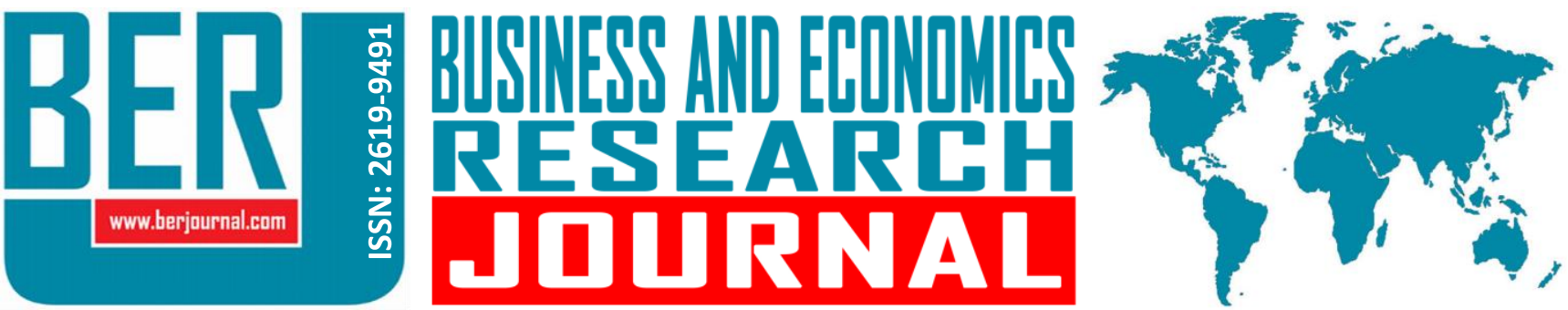 \\ Business and Economics Research Journal Vol. 13, No.1, $2022 \quad$ pp. 1-9 \\ doi: $10.20409 /$ berj.2022.358
}

\section{Location Choice under Spillovers}

\author{
Özgün Ekici ${ }^{\mathrm{a}}$, Bugra Caskurlu ${ }^{\mathrm{b}}$
}

Abstract: This paper introduces the location choice under spillovers game: A number of firms choose from among a number of alternative locations. A firm's payoff at some location is the sum of two factors: Its location-specific idiosyncratic payoff; and the positive spillover it receives, which is a function of the number of firms choosing the same location. The spillover function is location-specific and monotonically increasing. This game form can be viewed as an extension of the classic "battle of sexes" game. It can also be used to model real-life game-theoretic situations with network effects, such as when app users choose from alternative social media or instant messaging apps. In our main result, we show that the location choice under spillovers game is a potential game, and hence, it always admits a Nash equilibrium in pure strategies. We also show that: A Nash equilibrium outcome need not be Pareto efficient. An outcome that is Pareto efficient need not be a Nash equilibrium. And a Nash equilibrium is not necessarily a strong equilibrium.

Keywords: Pure strategy Nash equilibrium, Existence, Potential Games, Location Choice, Spillovers, Network Externalities

JEL: C72, D62

\section{Introduction}

The spatial huddling of firms in the same business is a common phenomenon. New York City and London are home to many financial businesses. Silicon Valley in the U.S. is home to the world's largest hightech corporations. Hollywood, which serves as the center of the U.S. film industry, is home to many film studios. In a similar vein, small businesses in a city also often spatially come together and serve from the same location. Furniture stores come together in furniture bazaars, textile firms operate from textile districts, and auto mechanics congregate in auto industrial zones.

Although laws and regulations may play a role, the force that drives the huddling of the firms in the same business is often positive spillovers. In Silicon Valley, high-tech firms learn from one another-for instance, when employees switch between jobs. In Hollywood, film producers benefit from having access to various film sets and a multitude of sector employees of all sorts. And if we take the furniture bazaar example: People who want to buy furniture value variety. In a furniture bazaar, dozens of furniture stores are huddled together, and buyers can see a great many choices in a short while and without incurring travel expenses to multiple locations. Therefore, a furniture bazaar acts as a "magnet" for furniture buyers, and thus it benefits all the stores in it.

a Asst. Prof., PhD., Ozyegin University, Faculty of Business, Department of Economics, Istanbul, Turkiye, ozgun.ekici@ozyegin.edu.tr (ORCID ID: 0000-0001-7053-4735)

b Asst. Prof., PhD., TOBB ETU, Faculty of Business, Department of Computer Engineering, Ankara, Turkiye, bcaskurlu@etu.edu.tr (ORCID ID: 0000-0002-4647-205X) 
With above applications in mind, we introduce in this paper a game form that studies the firms' location choices under spillovers. Specifically, we consider the following game form: There are $n$ firms that choose from among $m$ alternative locations. Firms' choices are driven by two factors. First, due to positive spillovers, firms value "huddling": If $s$ firms choose location $j$, each of these firms receives a payoff of $f_{j}(s)$ units, where $f_{j}$ is the monotonic positive spillover function associated with location $j$. Second, firms have idiosyncratic location preferences: When firm $i$ chooses location $j$, it receives an idiosyncratic location payoff of $x_{i j}$ units. Notice that all firms would huddle in one location if there were no idiosyncratic location payoffs. However, due to the idiosyncratic location payoffs, firms may spread over to multiple locations.

Although we frame it as a game of firms' location choices under spillovers, our game form can also be used to model some other real-life scenarios. For instance, imagine potential app users choosing from several related computer applications. It may be that the users choose from alternative social media applications, such as Twitter, Facebook, and Instagram. There are clearly network effects associated with social media applications since an app becomes more appealing to its users if it has more users. Indeed, if there were no idiosyncrasy in app preferences, users would be "huddling together" in a single app. But social media applications are not perfect substitutes, and there is heterogeneity in preferences across users and, for the same user, over time. Therefore, the market sustains multiple social media apps. Notice that a similar situation is at play when potential users choose from alternative instant messaging apps, such as WhatsApp, Messenger, and Viber.

It is worth mentioning that our game form can be viewed as the "battle of sexes" game extended to more than two players. Below, in Example 1, we present an illustration of the battle of sexes game, and we show that it can be obtained as an instance of the location choice under spillovers game. But one caution is in point: In Example 1, in the payoff matrix, if the payoffs $(1,1)$ on the bottom-left corner are replaced by $(0,0)$, the game may still be viewed as a "battle of sexes" situation, but it cannot be obtained as an instance of or our game form. In other words, the battle of sexes has no one standard formulation in the literature, and our game form should be viewed as an extension of its certain formulations.

Example 1: "Battle of Sexes" as a location choice under spillovers game

Woman

\begin{tabular}{cl|c|c|}
\multicolumn{1}{c}{} & \multicolumn{1}{c}{ Opera } & \multicolumn{1}{c}{ Football } \\
\cline { 3 - 4 } Man & Opera & 2,3 & 0,0 \\
\cline { 3 - 4 } & Football & 1,1 & 3,2 \\
\cline { 3 - 4 } & &
\end{tabular}

Consider the "battle of sexes" game above: A man and a woman (husband and wife) are to choose which event to attend on a Sunday evening. They do not like to be separated, but their favorite events are not the same. The man prefers the football match over the opera concert and vice versa for the woman. This game can be expressed as an instance of the location choice under spillovers game as follows:

- Each person's payoff depends on the kind of event (location) he or she attends and if he or she attends the event alone or with his or her partner.

- The man gets 0 units of payoff if he attends the opera concert and 1 unit of payoff if he attends the football match.

- The woman gets 1 unit of payoff if she attends the opera concert and 0 units of payoff if she attends the football match. 
- Each person gets an additional 2 units of payoff if he or she attends an event with his or her partner.

There are some parallels between our game form and congestion games, introduced by Rosenthal (1973). Like in our setting, in a congestion game, there is a set of players $(N)$ and a set of alternative facilities $(M)$, and players choose from these facilities. Also, as in our setting, each player's payoff at some facility is a function of the total number of users of that facility - these functions are called "congestion cost functions." In a congestion game, players choose facilities to minimize their incurred congestion costs. In two respects, the congestion game is more general than our game form:

- A player's set of admissible actions is an a priori given set of subsets of $M$. Therefore, in a congestion game, players can choose multiple facilities and they can be restricted in their choices - they can only choose from the a priori given player-specific combinations of facilities.

- There is no restriction on congestion cost functions. The congestion cost functions can be monotonically increasing/decreasing or non-monotonic. Notice that the huddling incentive can be modeled in a congestion game if the congestion functions are chosen to be monotonically decreasing.

But our game form differs from a congestion game in that, in our game form, players (firms) also have idiosyncratic preferences over facilities (locations). The theoretical findings of our paper are as follows:

In his paper, Rosenthal (1973) proved that every congestion game possesses a Nash equilibrium in pure strategies. He proved this result by the potential function argument. In a similar vein, in our main result, Theorem 1, we show that every location choice under spillovers game possesses a Nash equilibrium in pure strategies. Our proof technique is similar to the proof technique in Rosenthal (1973). We show that our game form admits a potential function, which implies the existence result. Since the location choice under spillovers is a finite potential game, a learning process based on better-response dynamics converges to a pure-strategy Nash equilibrium.

We then turn our attention to the theoretical properties of the pure-strategy Nash equilibrium outcomes. A natural question of interest is whether a Nash equilibrium outcome is always Pareto efficient. It turns out that it may not be. In Proposition 1, we show that a Nash equilibrium outcome need not be Pareto efficient, and a Pareto efficient outcome need not be a Nash equilibrium.

In our game form, each firm chooses a single location, and the location spillover functions are monotonically increasing. In a study related to ours, Holzman and Law-Yone (1997) considered the special case of congestion games under which, similar to in our setting, each player chooses a single facility, and congestion functions are monotonically increasing. Under these assumptions, they showed that the set of Nash equilibria coincides with the set of strong equilibria. Their result, however, does not hold in our setting. In Proposition 2, we show that in a location choice under spillovers game, a Nash equilibrium may not be a strong equilibrium.

The rest of the paper is organized as follows. Section 2 briefly mentions the related literature. Section 3 introduces the model (our game form). Section 4 introduces our notions. Section 5 presents our results. Section 6 concludes.

\section{Related Literature}

As explained above, our location choice under spillovers game relates to congestion games. Rosenthal (1973) introduced this game form, and he proved that in a congestion game, a pure-strategy Nash equilibrium always exists. A singleton congestion game is a congestion game in which each player is restricted to choosing a single facility. In singleton congestion games, Holzman and Law-Yone (1997) showed that if congestion functions are monotonic, the set of Nash equilibria coincides with the set of strong equilibria. Milchtaich (1996) considered the extension of congestion games in which, at facilities, players face playerspecific congestion functions. In this setting, he showed that a Nash equilibrium always exists if each player 
is restricted to choosing a single facility. There are also computational studies on congestion games in the literature. For studies on the price of anarchy, see Roughgarden and Tardos (2002), Christodoulou and Koutsoupias (2005), and Awerbuch et al. (2013). For studies on the computation of a Nash equilibrium, see Caragiannis et al. (2011), Chien and Sinclair (2011), and Harks and Timmermans (2017). For other studies on congestion games and its special instances, see Feldman and Tennenholtz (2010), Anshelevich et al. (2013), and Caskurlu et al. (2020a, 2020b, 2021).

As in our paper, some studies in the literature show the existence of pure-strategy Nash equilibrium in various game forms. One game form that always admits a pure-strategy Nash equilibrium is congestion games, as shown by Rosenthal (1973). For other studies on the existence of pure-strategy Nash equilibrium in different game forms, see Tasnádi (1999), Holard (2000), Lu (2007), Yamazaki (2008), Noguchi (2009), and Mallick (2011).

\section{Model}

The location choice under spillovers game $G$ is a four-tuple $\langle N, M, x, f\rangle$ :

- $N=\{1,2, \cdots, n\}$ is a set of firms (players).

- $M=\{1,2, \cdots, m\}$ is a set of locations (pure strategies).

- $x=\left(x_{i j}\right)_{i \in N, j \in M}$ is the matrix for firms' idiosyncratic location payoffs.

- $f=\left(f_{j}\right)_{j \in M}$ is the vector for the monotonic location spillover functions.

In this game, the players are the firms, each firm's strategy space is the set of locations, and each firm's goal is to maximize its payoff. Firm $i$ 's payoff when it chooses location $j$ is the sum of two factors: its idiosyncratic location payoff at location $j$, denoted by $x_{i j}$. And the positive spillover to firm $i$ at location $j$, equal to $f_{j}(s)$, where $f_{j}$ is the spillover function at location $j$ and $s$ is the number of firms that choose location $j$. We assume that the spillover function $f_{j}$ is monotonic, i.e., for $s_{1}>s_{2}, f_{j}\left(s_{1}\right) \geq f_{j}\left(s_{2}\right)$.

For the sake of shortness, when $\bar{N} \subseteq N$ is the subset of firms that choose location $j$, we use $f_{j}(\bar{N})$ (instead of $f_{j}(|\bar{N}|)$ to denote the positive spillover to a firm choosing location $j$. Thus, if $\bar{N}$ is the set of firms that choose location $j$, and if $i \in \bar{N}$, then the firm $i$ 's payoff is equal to $x_{i j}+f_{j}(\bar{N})$.

An allocation $\mu: N \rightarrow M$ is a function that maps each firm to its location choice. Let $\mathcal{M}$ denote the domain of allocations. Note that an allocation corresponds to a strategy profile since it fully specifies the firms' location choices.

Abusing notation, let $\mu_{j}$ denote the set of firms that choose location $j$ at allocation $\mu$. That is, $\mu_{j}=$ $\{i \in N \mid \mu(i)=j\}$.

Let $\pi_{i}(\mu)$ denote firm $i^{\prime}$ s payoff at allocation $\mu$. That is, $\pi_{i}(\mu)=x_{i j}+f_{j}\left(\mu_{j}\right)$ where $j=\mu(i)$.

\section{Notions}

A coalition $C$ is a non-empty subset of firms, i.e., $C \subseteq N$ and $C \neq \emptyset$.

A coalition $C$ blocks an allocation $\mu$ if, at $\mu$, a deviation by coalition $C$ leads to an allocation under which every coalition member is better off. In formal terms: A coalition $C$ blocks an allocation $\mu$ if there exists $\bar{\mu} \in \mathcal{M}$ such that for all $i \in N \backslash C, \bar{\mu}(i)=\mu(i)$, and for all $i \in C, \pi_{i}(\bar{\mu})>\pi_{i}(\mu)$. In the definition, note that, at $\mu$, when coalition members deviate, the induced allocation is $\bar{\mu}$, and at $\bar{\mu}$, every coalition member's payoff is higher.

An allocation $\mu$ is a Nash equilibrium if there exists no $i \in N$ such that $\{i\}$ blocks $\mu$. In words, if an allocation is a Nash equilibrium, it means a deviation by a firm never leads to an increase in the firm's payoff. 
An allocation $\mu$ is a strong equilibrium if there exists no coalition $C$ such that $C$ blocks $\mu$. In words, if an allocation is a strong equilibrium, it means no deviation by a subset of firms leads to an increase in the payoff of each of these firms.

Let $N E(G) \subseteq \mathcal{M}$ and $S E(G) \subseteq \mathcal{M}$, denote, in order, the sets of Nash equilibrium allocations and strong equilibrium allocations. By definition, $N E(G) \subseteq S E(G)$.

An allocation $\bar{\mu}$ Pareto dominates another allocation $\mu$ if under $\bar{\mu}$, every firm is at least as well off, and at least one firm is strictly better off. In more formal terms, the allocation $\bar{\mu}$ Pareto dominates $\mu$ if for all $i \in N, \pi_{i}(\bar{\mu}) \geq \pi_{i}(\mu)$, and for some $i \in N, \pi_{i}(\bar{\mu})>\pi_{i}(\mu)$.

An allocation $\mu$ is Pareto efficient if there exists no allocation that Pareto dominates $\mu$.

Let $P E(G) \subseteq \mathcal{M}$ denote the set of allocations that are Pareto efficient.

\section{Results}

In our main result (Theorem 1), we show that the location choice under spillovers game is a "potential game." Consequently, in this game form, the set of Nash equilibria in pure strategies is non-empty and coincides with the local optima of the associated potential function. Since our main result builds on the potential function argument, some background information is useful.

The concept of potential function is first mentioned in Monderer and Shapley (1996). But even if they coined this term, earlier, without calling it, Rosenthal (1973) used a potential function to show that every congestion game possesses a Nash equilibrium in pure strategies. In their paper, Monderer and Shapley (1996) introduced several notions of potential function. The one used in our paper is the "exact potential function," which we define next.

Consider a game in strategic form with $n$ players. Let $s=\left(s_{1}, s_{2}, \cdots, s_{n}\right)$ be a strategy profile. Let $S=$ $S_{1} \times S_{2} \times \cdots \times S_{n}$ be the domain of strategy profiles. Let $u_{i}(s)$ be player $i$ 's payoff under the strategy profile $S$.

A function $\varphi: S \rightarrow \mathbb{R}$ is an (exact) potential function if for every $s \in S, \bar{s}_{i} \in S_{i}$,

$$
\varphi\left(\bar{s}_{i}, s_{-i}\right)-\varphi(s)=u_{i}\left(\bar{s}_{i}, s_{-i}\right)-u_{i}(s) .
$$

A potential game is a game that admits a potential function.

In words: $\varphi$ is a potential function if a player's deviation at a strategy profile leads to an equal change in the value of the potential function and the deviating player's payoff.

For a strategy profile $s$, its "neighborhood" consists of strategy profiles that vary from $s$ in at most one player's strategy. That is, the neighborhood of $s$ consists of strategy profiles of the form $\left(\bar{s}_{i}, s_{-i}\right)$, where $\bar{s}_{i} \in S_{i}$ for some player $i$. In a potential game, the set of Nash equilibria coincides with the set of "local optima" of the potential function. By a local optimum, we mean a strategy profile $s$ such that in the neighborhood of $s$, the potential function's value is maximized at $s$. To see this, note that:

- If $s$ is a Nash equilibrium, then, by the definition of Nash equilibrium, for every strategy profile $\left(\bar{s}_{i}, s_{-i}\right)$ in the neighborhood of $s, u_{i}\left(\bar{s}_{i}, s_{-i}\right)-u_{i}(s) \leq 0$. Then, by the definition of the potential function $\varphi, \varphi_{i}\left(\bar{s}_{i}, s_{-i}\right)-\varphi_{i}(s) \leq 0$. But then, by definition, $s$ is a local optimum of the potential function $\varphi$.

- If $s$ is a local optimum of the potential function $\varphi$, then, by definition of local optimum, for every strategy profile $\left(\bar{s}_{i}, s_{-i}\right)$ in the neighborhood of $s, \varphi_{i}(s)-\varphi_{i}\left(\bar{s}_{i}, s_{-i}\right) \geq 0$. Then, by the definition of the potential function $\varphi, u_{i}\left(\bar{s}_{i}, s_{-i}\right)-u_{i}(s) \leq 0$. But then, by definition, $s$ is a Nash equilibrium.

In a potential game, if we begin with an arbitrary strategy profile, whenever a player takes a deviation that makes the player better off, the value of the potential function increases. Therefore, in a finite potential game, the players' myopic better-response dynamics always converge to a Nash equilibrium outcome. For 
further discussions on better-response dynamics in potential games, see Monderer and Shapley (1996) and Voorneveld (2000).

We are now ready to present the main result of our paper.

Theorem 1: The location choice under spillovers game is a potential game. Therefore, the set of Nash equilibria in pure strategies coincides with the local optima of the associated potential function. Furthermore, since the game is finite, the set of Nash equilibria in pure strategies is non-empty.

\section{Proof}

For some allocation $\mu$, let:

- $x_{j}\left(\mu_{j}\right)=\sum_{i \in \mu_{j}} x_{i j}$

- $F_{j}\left(\mu_{j}\right)=f_{j}(0)+f_{j}(1)+\cdots+f_{j}\left(\mu_{j}\right)$

Above, $x_{j}\left(\mu_{j}\right)$ is the sum of the idiosyncratic location payoffs to firms that choose location $j$ at $\mu$. But the function $F_{j}\left(\mu_{j}\right)$ does not have a meaningful interpretation. In particular, it is not equal the sum of positive spillovers to firms that choose location $j$ at $\mu$. (That sum would be equal to $f_{j}\left(\mu_{j}\right) \cdot\left|\mu_{j}\right|$.) The function $F_{j}\left(\mu_{j}\right)$ is simply an artifact of definition that we will use in the definition of the game's potential function.

A potential function maps players' strategies to real numbers. In a location choice under spillovers game, an allocation corresponds to a strategy profile since it specifies every firm's location choice. Therefore, in this game form, a potential function should map allocations to real numbers. $F_{j}\left(\mu_{j}\right)$.

Let the function $\varphi: \mathcal{M} \rightarrow \mathbb{R}$ be defined as follows: $\varphi(\mu)=\sum_{j \in M} \varphi_{j}(\mu)$ where $\varphi_{j}(\mu)=x_{j}\left(\mu_{j}\right)+$

We will show that $\varphi$ is a potential function of the location choice under spillovers game. To show this, we will show that a deviation by a firm at some allocation leads to an equal change in the function's value and the deviating firm's payoff.

At allocation $\mu$, let firm $i$ deviate from location $k$ to $l$. Let $\bar{\mu}$ be the allocation induced. Then, firm $i$ 's payoff changes from $\pi_{i}(\mu)=x_{i k}+f_{k}\left(\mu_{k}\right)$ to $\pi_{l}(\bar{\mu})=x_{i l}+f_{l}\left(\bar{\mu}_{l}\right)$. Thus:

$$
\pi_{i}(\bar{\mu})-\pi_{i}(\mu)=\left(x_{i l}+f_{l}\left(\bar{\mu}_{l}\right)\right)-\left(x_{i k}+f_{k}\left(\mu_{k}\right)\right)
$$

The change in $\varphi^{\prime}$ s value is $\varphi(\bar{\mu})-\varphi(\mu)=\sum_{j \in M} \varphi_{j}(\bar{\mu})-\sum_{j \in M} \varphi_{j}(\mu)$. For $j \notin\{k, l\}$, note that $\mu_{j}=$ $\bar{\mu}_{j}$. Then, for $j \notin\{k, l\}, \varphi_{j}(\mu)=\varphi_{j}(\bar{\mu})=x_{j}\left(\mu_{j}\right)+F_{j}\left(\mu_{j}\right)$. Thus, we get:

$$
\begin{aligned}
& \varphi(\bar{\mu})-\varphi(\mu)=\left(\varphi_{k}(\bar{\mu})+\varphi_{l}(\bar{\mu})\right)-\left(\varphi_{k}(\mu)+\varphi_{l}(\mu)\right) \\
& =\left(\varphi_{k}(\bar{\mu})-\varphi_{k}(\mu)\right)+\left(\varphi_{l}(\bar{\mu})-\varphi_{l}(\mu)\right)
\end{aligned}
$$

When we switch from $\mu$ to $\bar{\mu}$, everything remains the same at locations $k$ and $l$, with the exception that firm $i$ is dropped from location $k$ and added to location $l$. Then, using the definition of the function $\varphi_{j}$, we obtain the following:

$$
\varphi_{k}(\bar{\mu})-\varphi_{k}(\mu)=-x_{i k}-f_{k}\left(\mu_{k}\right) \text { and } \varphi_{l}(\bar{\mu})-\varphi_{l}(\mu)=x_{i l}+f_{l}\left(\bar{\mu}_{l}\right)
$$

Thus:

$$
\varphi(\bar{\mu})-\varphi(\mu)=\left(x_{i l}+f_{l}\left(\bar{\mu}_{l}\right)\right)-\left(x_{i k}+f_{k}\left(\mu_{k}\right)\right)
$$

Therefore, we found that:

$$
\varphi(\bar{\mu})-\varphi(\mu)=\pi_{i}(\bar{\mu})-\pi_{i}(\mu)
$$

As desired, we found that a deviation by a firm leads to an equal change in the value of the function $\varphi$ and the deviating firm's payoff. This shows that the location choice under spillovers game is a potential game, and the function $\varphi$ is its potential function. Therefore, the set of Nash equilibria in pure strategies 
coincides with the local optima of the potential function. Since the game is finite, the set of Nash equilibria in pure strategies is also non-empty.

Theorem 1 shows that a location choice under spillovers game always admits a Nash equilibrium outcome. A natural question that ensues is whether a Nash equilibrium outcome is always Pareto efficient. The answer turns out to be negative. The converse statement is also not true. We present this result in Proposition 1.

Proposition 1: In a location choice under spillovers game, a Nash equilibrium outcome need not be Pareto efficient, and an outcome that is Pareto efficient need not be a Nash equilibrium, i.e., we may have $N E(G) \backslash P E(G) \neq \varnothing$ and $P E(G) \backslash N E(G) \neq \varnothing$.

\section{Proof}

For $N E(G) \backslash P E(G) \neq \varnothing$, see Example 2. For $P E(G) \backslash N E(G) \neq \emptyset$, see Example 3.

\section{Example 2}

Suppose that there are two firms and two locations and both firms prefer location 1 to 2 . Suppose that there is a sufficiently large positive spillover when the two firms are together. Thus, both firms prefer to be in the same location, even if it means they are at location 2. For specificity, we have:

- $x_{11}=x_{21}=1$ and $x_{12}=x_{22}=0$

- $f_{j}(0)=0, f_{j}(1)=1, f_{j}(2)=100$ for $j=1,2$

Consider these two allocations:

- $\bar{\mu}$ such that $\bar{\mu}(1)=\bar{\mu}(2)=1$

- $\mu$ such that $\mu(1)=\mu(2)=2$

The allocation $\mu$ is a Nash equilibrium: if a single firm deviates to location 1 , its payoff decreases from 100 to 2 . But $\mu$ is not Pareto efficient: $\bar{\mu}$ Pareto dominates $\mu$.

\section{Example 3}

Suppose that there are two firms and two locations, firm 1 prefers location 1 to 2 , and firm 2 prefers location 2 to 1 . Suppose that for each firm, the idiosyncratic payoff at its favorite location is sufficiently high so that the firm prefers to be at its favorite location, even if it will be alone there. For specificity, consider the following parameterization of the game:

- $x_{11}=100, x_{12}=0, x_{21}=0, x_{22}=100$

- $f_{j}(0)=0, f_{j}(1)=1, f_{j}(2)=2$ for $j=1,2$

Consider the allocation $\mu$ such that $\mu(1)=\mu(2)=1$. The allocation $\mu$ is Pareto efficient because, at any other allocation, firm 1's payoff is lower. But $\mu$ is not a Nash equilibrium because, at $\mu$, if firm 2 deviates to location 2, its payoff increases from 2 to 101.

Holzman and Law-Yone (1997) considered a game form that has some parallels to our location choice under spillovers game. As in our setting, each player chooses a facility from a subset of alternative facilities. But unlike in our setting, players suffer when they come together. Facilities chosen by too many players become "congested" and less valuable to users. As in our setting, they study this game form under the monotonicity assumption. They show that when congestion cost functions are monotonic, the set of Nash equilibria coincides with the set of strong equilibria. In Proposition 2, we show that this result does not hold in our setting. 
Proposition 2: In a location choice under spillovers game, a Nash equilibrium may not be a strong equilibrium, i.e., we may have $N E(G) \backslash S E(G) \neq \emptyset$.

\section{Proof}

Consider the allocations $\mu$ and $\bar{\mu}$ in Example 2. As explained there, $\mu$ is a Nash equilibrium. But notice that the coalition $\{1,2\}$ blocks $\mu$ : when firms 1 and 2 both deviate to location 1 , the allocation induced is $\bar{\mu}$, and both firms' payoffs are higher at $\bar{\mu}$. Thus, the allocation $\mu$ is a Nash equilibrium but not a strong equilibrium.

\section{Conclusion}

In this paper, we introduced the location choice under spillovers game. In this game form, a number of firms choose from a number of alternative locations, and each firm's payoff at some location is the sum of the firm's location-specific idiosyncratic payoff and the spillover the firm receives at that location. This game form is similar to a congestion game since the payoff of a player (firm) at some facility (location) is a function of the number of players that choose the facility. But unlike in a congestion game, a player's payoff at some facility also includes the player's location-specific idiosyncratic payoff.

In our main result, we show that the location choice under spillovers is a potential game. Thus, there always exists a Nash equilibrium in pure strategies. Since the game is finite, a process based on betterresponse dynamics always converges to a pure-strategy Nash equilibrium. In other words, beginning with some random allocation, if firms take payoff-increasing deviations one at a time, the process ultimately yields a Nash equilibrium outcome. We also showed some drawbacks associated with a Nash equilibrium. In particular, we showed that a Nash equilibrium may not be Pareto efficient or a strong equilibrium.

Our findings have the following policy consequences: On one hand, the fact that a process based on the better-response dynamics converges to a pure-strategy Nash equilibrium indicates that a stable spatial configuration of firms can be attained in a free market, without any guidance from the government. On the other hand, the fact that not all Nash equilibria are Pareto efficient hints that government interference may be useful to achieve superior equilibrium outcomes. For instance, if doing so is efficient, the government may incentivize firms in the defense industry to congregate in a particular region of the country or firms in the textile industry to congregate in a specific district of a city.

In a congestion game, when each player chooses a single facility, Milchtaich (1996) showed that a pure-strategy Nash equilibrium always exists even if players face player-specific congestion functions at facilities. One open research question is whether the same result holds in our setting. That is, future research may explore whether the existence of a pure-strategy Nash equilibrium is guaranteed if at some location firms face firm-specific spillover functions.

\section{Declarations and Disclosures}

Ethical Responsibilities of Authors: The authors of this article confirm that their work complies with the principles of research and publication ethics.

Conflicts of Interest: No potential conflict of interest was reported by the authors.

Funding: The authors received no financial support for the preparation and/or publication of this article.

Author Contributions: The authors confirm contribution to the article as follows: Conceptualization and design, Ö. Ekici and B. Caskurlu; writing the first draft of the manuscript, Ö. Ekici and B. Caskurlu; review and editing, Ö. Ekici and B. Caskurlu. The manuscript/article was read and approved by all the authors, and all authors accepted responsibility for their article.

Plagiarism Checking: This article was screened for potential plagiarism using a plagiarism screening program. 


\section{References}

Anshelevich, E., Caskurlu, B., \& Hate, A. (2013). Partition equilibrium always exists in resource selection games. Theory of Computing Systems, 53(1), 73-85.

Awerbuch, B., Azar, Y., \& Epstein, A. (2013). The price of routing unsplittable flow. SIAM Journal on Computing, 42(1), 160-177.

Caragiannis, I., Fanelli, A., Gravin, N., \& Skopalik, A. (2011). Efficient computation of approximate pure Nash equilibria in congestion games. 2011 IEEE 52nd Annual Symposium on Foundations of Computer Science, pp.532-541. https://doi.org/10.1109/FOCS.2011.50

Caskurlu, B., Ekici, Ö., \& Kizilkaya, F. E. (2020a). On existence of equilibrium under social coalition structures. Lecture Notes in Computer Science (Including Subseries Lecture Notes in Artificial Intelligence and Lecture Notes in Bioinformatics). https://doi.org/10.1007/978-3-030-59267-7_23

Caskurlu, B., Ekici, Ö., \& Kizilkaya, F. E. (2020b). On efficient computation of equilibrium under social coalition structures. Turkish Journal of Electrical Engineering \& Computer Sciences, 28(3), 1686-1698.

Caskurlu, B., Ekici, Ö., \& Kizilkaya, F. E. (2021). On singleton congestion games with resilience against collusion. (pp. 3748). https://doi.org/10.1007/978-3-030-89543-3_4

Chien, S., \& Sinclair, A. (2011). Convergence to approximate Nash equilibria in congestion games. Games and Economic Behavior, 71(2), 315-327.

Christodoulou, G., \& Koutsoupias, E. (2005). The price of anarchy of finite congestion games. Proceedings of the ThirtySeventh Annual ACM Symposium on Theory of Computing - STOC '05, 67. https://doi.org/10.1145/1060590.1060600

Feldman, M., \& Tennenholtz, M. (2010). Structured coalitions in resource selection games. ACM Transactions on Intelligent Systems and Technology, 1(1), 1-21.

Harks, T., \& Timmermans, V. (2017). Equilibrium computation in atomic splittable singleton congestion games. In: F. Eisenbrand \& J. Koenemann (Eds.), Integer Programming and Combinatorial Optimization. IPCO 2017. Lecture Notes in Computer Science, (pp 442-454). Springer, Cham.

Hollard, G. (2000). On the existence of a pure strategy Nash equilibrium in group formation games. Economics Letters, 66(3), 283-287.

Holzman, R., \& Law-Yone, N. (1997). Strong equilibrium in congestion games. Games and Economic Behavior, 21(1-2), 85-101.

Lu, H. (2007). On the existence of pure-strategy Nash equilibrium. Economics Letters, 94(3), 459-462.

Mallick, I. (2011). On the existence of pure strategy Nash equilibria in two-person discrete games. Economics Letters, $111(2), 144-146$.

Milchtaich, I. (1996). Congestion games with player-specific payoff functions. Games and Economic Behavior, 13(1), 111124.

Monderer, D., \& Shapley, L. S. (1996). Potential games. Games and Economic Behavior, 14(1), 124-143.

Noguchi, M. (2009). Existence of Nash equilibria in large games. Journal of Mathematical Economics, 45(1-2), 168-184.

Rosenthal, R. W. (1973). A class of games possessing pure-strategy Nash equilibria. International Journal of Game Theory, 2, 65-67.

Roughgarden, T., \& Tardos, É. (2002). How bad is selfish routing? Journal of the ACM, 49(2), 236-259.

Tasnádi, A. (1999). Existence of pure strategy Nash equilibrium in Bertrand-Edgeworth oligopolies. Economics Letters, 63(2), 201-206.

Voorneveld, M. (2000). Best-response potential games. Economics Letters, 66(3), 289-295.

Yamazaki, T. (2008). On the existence and uniqueness of pure-strategy Nash equilibrium in asymmetric rent-seeking contests. Journal of Public Economic Theory, 10(2), 317-327. 
This Page Intentionally Left Blank 